\title{
Análise de tendência temporal dos casos confirmados de tuberculose no Brasil de 2011 a 2020
}

\author{
Temporal trend analysis of confirmed tuberculosis cases in Brazil from 2011 to 2020 \\ Análisis de tendencia temporal de casos confirmados de tuberculosis en Brasil de 2011 a 2020
}

\begin{abstract}
RESUMO
Objetivo: Analisar a tendência temporal de casos confirmados e notificados de Tuberculose (TB) no Brasil de 2011 à 2020, segundo características demográficas, socioeconômicas e regionais. Método: Trata-se de um estudo de série temporal com dados oriundos do Sistema de Informação de Agravos de Notificação (SINAN). A análise de tendência foi obtida utilizando-se regressão linear simples, com nível de significância de 5\%. Para o processamento dos dados e análises estatísticas, utilizou-se o Software for Statistics and Data Science (Stata). Resultado: Evidenciou-se uma tendência de estabilidade dos casos de TB no Brasil. Houve aumento significativo dos casos entre homens, pardos, pessoas acima de 55 anos, residentes na região norte do país, bem como o expressivo aumento entre pessoas alcoólicas e diabéticas. Conclusão: Apesar de sugerirem a melhora das estratégias de vigilância, prevenção e controle da doença, demandam ações intersetoriais de enfrentamento da TB.
\end{abstract}

DESCRITORES: Tuberculose; Epidemiologia; Saúde Pública; Controle de Doenças Transmissíveis.

\section{ABSTRACT}

Objective: To analyze the time trend of confirmed and reported cases of Tuberculosis (TB) in Brazil from 2011 to 2020, according to demographic, socioeconomic and regional characteristics. Method: This is a time series study with data from the Sistema de Informação de Agravos de Notificação (SINAN). Trend analysis was obtained using simple linear regression, with a 5\% significance level. The Software for Statistics and Data Science (Stata) was used for data processing and statistical analysis. Results: A tendency toward stability of TB cases in Brazil was evident. There was a significant increase in cases among males, mixed race, persons over 55 years of age, residents of the northern region of the country, as well as a significant increase among alcoholics and diabetics. Conclusion: Despite suggesting the improvement of strategies for surveillance, prevention and control of the disease, they demand intersectoral actions to confront TB.

DESCRIPTORS: Tuberculosis; Epidemiology; Public Health; Communicable Disease Control.

\section{RESUMEN}

Objetivo: Analizar la tendencia temporal de los casos confirmados y notificados de Tuberculosis (TB) en Brasil de 2011 a 2020, según las características demográficas, socioeconómicas y regionales. Metodo: Se trata de un estudio de series temporales con datos del Sistema de Informação de Agravos de Notificação (SINAN). El análisis de la tendencia se obtuvo mediante una regresión lineal simple con un nivel de significación del 5\%. Para el tratamiento de los datos y el análisis estadístico se utilizó el programa informático de estadística y ciencia de los datos (Stata). Resultados: Se evidenció una tendencia a la estabilidad de los casos de TB en Brasil. Hubo un aumento significativo de casos entre los hombres, la raza mixta, las personas mayores de 55 años, los residentes de la región norte del país, así como un aumento significativo entre los alcohólicos y los diabéticos. Conclusión: Además de sugerir la mejora de las estrategias de vigilancia, prevención y control de la enfermedad, se requieren acciones interdisciplinarias para hacer frente a la TB.

Descriptores: Tuberculosis; Epidemiología; Salud Pública; Control de Enfermedades Transmisibles.

RECEBIDO EM: 11/10/2021 APROVADO EM: 01/12/2021

\author{
Fernanda Lara Pereira de Souza \\ Enfermeira graduada pela Universidade do Estado de Minas Gerais - Unidade de Passos. \\ ORCID: 0000-0002-3553-7401
}

\section{Lauren Matozinhos Clark}

Graduanda em Enfermagem pela Universidade do Estado de Minas Gerais - Unidade Passos.

ORCID: 0000-0001-6354-9699

\section{Elton Junio Sady Prates}

Graduando em Enfermagem pela Universidade Federal de Minas Gerais.

ORCID: 0000-0002-5049-186X 


\section{Maria Luiza Sady Prates}

Graduanda em Enfermagem pela Universidade Federal de Minas Gerais.

ORCID: 0000-0001-6199-7092

\section{Josely Pinto de Moura}

Docente do Curso de Enfermagem e Ciências Biológicas (Bacharelado) da Universidade do Estado de Minas Gerais - Unidade Passos. Doutora pelo Programa Interunidades de Doutoramento em Enfermagem - USP/ SP/ RP, Mestre em Enfermagem Fundamental pela USP, Especialista em Controle de Infecção Hospitalar (USP), especialista em Administração Hospitalar (UNAERP), Graduada em Enfermagem pela Faculdade de Enfermagem e Obstetrícia de Passos.

ORCID: 0000-0002-3856-4181

\section{INTRODUÇÃO}

A tuberculose (TB) é uma doença transmissível infecciosa mais antiga da humanidade e, mesmo tratável, é considerada um problema de saúde pública global e uma das doenças que mais leva à morte. 1,2

A vulnerabilidade à TB é influenciada por determinantes biológicos que afetam a resposta imune ao Mycobacterium tuberculosis, como ocorre em crianças pequenas e pacientes com comorbidades, como viver com HIV ou diabetes. 3,4

A fragilidade à TB também é influenciada por determinantes sociais intimamente ligados a situação de pobreza o que gere a exposição dos indivíduos a um maior risco de contato com M. tuberculosis e consequente adoecimento devido às condições precárias de moradia, desnutrição, condições inadequadas de trabalho e falta de acesso aos serviços de saúde. Além disso, os pacientes com TB enfrentam a perda de produtividade no trabalho e os altos custos associados ao diagnóstico e tratamento da TB. 3,4

O ano de 2015 tornou-se um marco na história da TB, quando a Organização Mundial da Saúde (OMS) propôs uma Estratégia Global pelo Fim da Tuberculose.6 Ademais, outros documentos no âmbito global destacaram compromissos relacionados à visão de um mundo livre da doença, com destaque para a Declaração de Moscou (2017) e a Declaração da Reunião de Alto Nível (2018).4

As perspectivas globais pertinentes às políticas de TB estão centradas na sustentabilidade das ações de controle da doença, por meio de financiamento adequado, comprometimento político de alto nível e engajamento multissetorial.5 A fim de contribuir com as metas de redução de $90 \%$ na taxa de incidência; redução de $95 \%$ no

\section{A vulnerabilidade \\ à TB é influenciada por determinantes}

biológicos

que afetam a

resposta imune ao

Mycobacterium

tuberculosis, como

ocorre em crianças

pequenas e pacientes

com comorbidades,

como viver com

HIV ou diabetes

número de mortes relacionadas à doença; e permitir que nenhuma pessoa com TB, bem como seus familiares, enfrentem catas- tróficos custos decorrentes da infecção.3,4 Ainda, ressalta-se a importância da organização e da dinâmica dos serviços de saúde nas ações de controle da TB, como também na forma em que os serviços se organizam para detectar e tratar os casos. 2

Em 2020, a OMS divulgou um relatório sobre o monitoramento dos progressos relacionados às metas globais da $\mathrm{TB}$ e a implementação da Declaração Política da Reunião de Alto Nível. O documento chama a atenção para o status atual dos marcos intermediários das metas da Estratégia Global pelo Fim da Tuberculose estabelecidos para o período de 2015 a 2020, que não foram alcançados. 5

Nessa perspectiva, objetivou-se analisar a tendência temporal de casos confirmados e notificados de TB no Brasil de 2011 a 2020.

\section{MÉTODO}

Trata-se de um estudo de série temporal. Os dados foram obtidos por meio do Sistema de Informação de Agravos de Notificação (SINAN) e foram exportados do Departamento de Informática do Sistema Único de Saúde (Datasus), via TABNET, um aplicativo desenvolvido pelo DATASUS que disponibiliza informações sobre saúde pública. Dessa forma, os dados foram coletados no dia 8 de setembro de 2021, sendo esses extraídos do ano de 2011 a 2020.

A análise de tendência da série temporal do indicador de TB foi estratificada segundo Sexo (masculino; feminino); Faixa etária (anos: 0-14; 15-24; 25-34; 35-44; 45-54; 55-64; 65 ou mais); Nível de Escolaridade (analfabeto; $1^{\mathrm{a}}$ a $4^{\mathrm{a}}$ série incom- 


\section{artigo}

Souza, F. L. P., Clark, L. M., Prates, E. J. S., Prates, M. L. S., Moura, J. P.

Análise de tendência temporal dos casos confirmados de tuberculose no Brasil de 2011 a 2020

pleta; $4^{\mathrm{a}}$ série completa do EF; $5^{\mathrm{a}}$ a $8^{\mathrm{a}}$ série incompleta; Ensino fundamental; Ensino médio incompleto; Ensino médio completo; Educação superior; Educação superior completa); Raça/cor da pele (branca; preta; amarela; parda; indígena); Infecção por Vírus da Imunodeficiência Humana (HIV); síndrome de imunodeficiência adquirida (AIDS); diabetes mellitus; doença mental; tabagismo; alcoolismo; uso de drogas ilícitas; casos notificados no Brasil e regiões.

Para avaliar a tendência temporal aplicou-se o modelo de regressão linear simples cuja variável (Yi) foi o local e a variável independente $(\mathrm{Xi})$ dada pelo tempo (ano do levantamento). O sinal negativo do coeficiente angular $(\beta)$ da reta ajustada pelo modelo indicou que a relação entre o indicador e o tempo era decrescente, enquanto o valor do coeficiente angular positivo representou o aumento médio anual.

Como medida de adequação do modelo utilizou-se a análise de resíduo e o R-quadrado que indica o percentual de explicação dos valores da variável dependente. Adotou-se o nível de significância de 5\%. Para o processamento dos dados e análises estatísticas, utilizou-se o Software for Statistics and Data Science (Stata) versão 16.

Foram utilizados exclusivamente dados secundários, que não permitem a identificação dos sujeitos, minimizando assim os potenciais riscos aos participantes. Todos os dados foram analisados segundo os princípios éticos estabelecidos nas Resoluções no 466/12, e 510/16 do Conselho Nacional de Saúde.

\section{RESULTADOS}

Evidenciou-se uma tendência de esta-

bilidade dos casos de TB no Brasil, sendo registrados 87.813 casos em 2011 e 86.166 em 2020. Dentre os casos registrados, observou-se uma tendência significativa $(\mathrm{p}<$ $0,05)$ de aumento entre o sexo masculino, raça/cor da pele parda, residentes da região Norte do país, pessoas na faixa etária de 15 a 24 anos e acima de 55 anos de idade (tabela 1).

Verificou-se, ainda, uma tendência significativa $(\mathrm{p}<0,05)$ de declínio dos casos entre os níveis de escolaridade mais baixos e aumento significativo de casos entre pessoas com segundo grau completo (tabela 2). Ademais, constatou-se uma tendência significativa $(\mathrm{p}<0,05)$ de expressivo aumento de casos de TB entre pessoas alcoólicas e portadores de diabetes (tabela 3).

\section{DISCUSSÃO}

Tabela 1 - Tendência temporal dos casos confirmados e notificados de tuberculose segundo variáveis sexo, faixa etária, raça/cor da pele, Brasil, 2010-2020.

\begin{tabular}{|c|c|c|c|c|c|c|c|c|c|c|c|c|}
\hline \multirow{2}{*}{ Variável } & \multicolumn{10}{|c|}{ Ano } & \multirow{2}{*}{ p - valor } & \multirow{2}{*}{$\mathbf{B}^{*}$} \\
\hline & 2011 & 2012 & 2013 & 2014 & 2015 & 2016 & 2017 & 2018 & 2019 & 2020 & & \\
\hline \multicolumn{13}{|l|}{ Sexo } \\
\hline Masculino & 59.136 & 58.269 & 58.267 & 58.077 & 59.004 & 59.680 & 63.707 & 66.250 & 67.687 & 60.722 & 0.0201 & 834.4 \\
\hline Feminino & 28.676 & 27. 902 & 27.933 & 27.131 & 26.443 & 26.524 & 27.064 & 28.462 & 28.958 & 25.438 & 0.3780 & -116.5 \\
\hline \multicolumn{13}{|l|}{ Faixa E tária } \\
\hline 0 a 14 anos & 2.756 & 2.547 & 2.658 & 2.385 & 2.275 & 2.336 & 2.430 & 2.722 & 2.886 & 2.134 & 0.5661 & -16.41 \\
\hline 15 a 24 anos & 14.334 & 14.388 & 14.173 & 13.928 & 14.624 & 15.076 & 16.452 & 17.421 & 17.507 & 14.725 & 0.0327 & 300.7 \\
\hline 25 a 34 anos & 21.215 & 20.723 & 20.539 & 20.482 & 20.160 & 20.072 & 21.174 & 21.917 & 22.395 & 20.090 & 0.5008 & 63.37 \\
\hline 35 a 44 anos & 17.262 & 16.872 & 17.062 & 16.778 & 16.623 & 16.975 & 17.509 & 18.493 & 18.723 & 16.647 & 0.2248 & 103.7 \\
\hline 45 a 54 anos & 15.039 & 14.792 & 14.490 & 14.264 & 13.941 & 13.740 & 14.054 & 14.622 & 14.678 & 12.957 & 0.0715 & -119.4 \\
\hline 55 a 64 anos & 9.392 & 9.446 & 9.582 & 9.817 & 10.068 & 10.021 & 10.610 & 10.830 & 11.202 & 9.794 & 0.0159 & 148.3 \\
\hline $65 \mathrm{ou}+$ & 7.736 & 7.314 & 7.591 & 7.476 & 7.745 & 7.977 & 8.532 & 8.704 & 9.187 & 8.033 & 0.0110 & 149.9 \\
\hline \multicolumn{13}{|c|}{ Raça/Cor da Pele } \\
\hline Branca & 29.142 & 29.597 & 28.650 & 27.937 & 27.086 & 26.716 & 26.332 & 27.182 & 27.963 & 27.628 & 0.0407 & -227.8 \\
\hline Preta & 11.524 & 11.966 & 11.787 & 11.206 & 11.154 & 10.871 & 11.027 & 11.444 & 12.244 & 12.632 & 0.3934 & 56.86 \\
\hline Am arela & 743 & 709 & 705 & 663 & 610 & 582 & 422 & 537 & 552 & 720 & 0.1237 & -17.55 \\
\hline Parda & 35.293 & 37.644 & 37.882 & 38.682 & 38.535 & 39.427 & 40.428 & 43.665 & 46.289 & 47.358 & 0.0000 & 1.237 \\
\hline Indigena & 884 & 994 & 883 & 945 & 870 & 993 & 931 & 912 & 936 & 929 & 0.7912 & 1.363 \\
\hline
\end{tabular}

Fonte: Ministério da Saúde/SVS - Sistema de Informação de Agravos de Notificação - Sinan Net Notas: Os dados foram coletados no dia 8 de setembro de 2021.

O cálculo do p-valor foi feito pela regressão linear.

Foram considerados, como estatisticamente significativos, os casos em que $\mathrm{p}$-valor $<0.05$

* Indica o coeficiente de variação angular. 
Tabela 2 - Tendência temporal dos casos confirmados e notificados de tuberculose segundo variáveis escolaridade e distribuição dos casos pelas regiões do país, Brasil, 2011-2020.

\begin{tabular}{|c|c|c|c|c|c|c|c|c|c|c|c|c|}
\hline \multirow{2}{*}{ Variável } & \multicolumn{10}{|c|}{ Ano } & \multirow{2}{*}{ p - valor } & \multirow{2}{*}{$\mathrm{B}^{*}$} \\
\hline & 2011 & 2012 & 2013 & 2014 & 2015 & 2016 & 2017 & 2018 & 2019 & 2020 & & \\
\hline \multicolumn{13}{|l|}{ Escolaridade } \\
\hline Analfabeto & 4248 & 4.195 & 3.882 & 4.158 & 3243 & 3.668 & 3.756 & 4.367 & 4.133 & 3.168 & 0.2899 & -51.57 \\
\hline $1^{2}$ a $4^{2}$ série incompleta do EF & 11.677 & 13.306 & 12.420 & 11.771 & 11.107 & 10.921 & 10.430 & 10.351 & 10.150 & 8.407 & 0.0005 & -400.4 \\
\hline $4^{2}$ série compleła do $\mathbb{F}$ & 5.542 & 5.370 & 4.898 & 4.523 & 4.399 & 4.104 & 3.970 & 4.161 & 4.021 & 4.009 & 0.0003 & -171.6 \\
\hline $5^{2}$ a $8^{2}$ série incompleta do EF & 12.318 & 17.374 & 17.172 & 16.895 & 16.839 & 17.093 & 16.533 & 17.670 & 18.831 & 12623 & 0.7293 & 88.49 \\
\hline Ers ino fundamertal completo & 4.549 & 4.724 & 4255 & 4.594 & 4245 & 4.343 & 4.553 & 4.618 & 5.287 & 5.373 & 0.0509 & 79.67 \\
\hline Ersino médio incompleto & 5.165 & 9.876 & 9.898 & 10.002 & 10.024 & 10.268 & 10.675 & 11.463 & 11.992 & 5.737 & 0.4982 & 1821 \\
\hline Ers ino médio completo & 6.350 & 6.658 & 7.140 & 7.112 & 7.149 & 7.138 & 7.457 & 7.784 & 8.555 & 9.233 & 0.0002 & 283.4 \\
\hline Educação superior incompleta & 1.904 & 1.985 & 2.055 & 2.183 & 2.164 & 2.192 & 2.368 & 2550 & 2688 & 1.255 & 0.7905 & 12.10 \\
\hline Educacão superior completa & 1.923 & 2.101 & 2242 & 2313 & 2309 & 2316 & 2476 & 2.432 & 2853 & 2220 & 0.0239 & 56.86 \\
\hline \multicolumn{13}{|l|}{ Região do país } \\
\hline Norte & 8.742 & 8.566 & 8.877 & 8.776 & 9.013 & 9.365 & 10.157 & 10.389 & 11.690 & 10.529 & 0.0006 & 3030 \\
\hline Nordeste & 24.385 & 23.729 & 23.073 & 22.390 & 22202 & 22.545 & 23.803 & 25.134 & 25.058 & 21.882 & 0.9436 & 10.07 \\
\hline Sudeste & 39.490 & 38.412 & 38.463 & 38.578 & 39.193 & 39.543 & 41.428 & 42.773 & 42848 & 38.799 & $0.07 / 2$ & 3350 \\
\hline Sul & 11.300 & 11.168 & 11.268 & 11.174 & 11.028 & 10.678 & 11.235 & 11.867 & 12.453 & 10.828 & 0.4488 & 45.98 \\
\hline Centro-Oeste & 3.896 & 4.308 & 4.529 & 4295 & 4.018 & 4.076 & 4.155 & 4.557 & 4.605 & 4.128 & 0.4054 & 2390 \\
\hline Brasil & 87.813 & 86.183 & 86208 & 85213 & 85.452 & 86.207 & 90.776 & 94.720 & 96.655 & 86.186 & 0.1162 & 718.0 \\
\hline
\end{tabular}

Fonte: Ministério da Saúde/SVS - Sistema de Informação de Agravos de Notificação - Sinan Net Notas: Os dados foram coletados no dia 8 de setembro de 2021.

Foram considerados, como estatisticamente significativos, os casos em que p-valor $<0.05$.

* Indica o coeficiente de variação angular.

Tabela 3 - Tendência temporal dos casos confirmados e notificados de tuberculose segundo variáveis comorbidades e fatores de risco associados, Brasil, 2011-2020.

\begin{tabular}{|c|c|c|c|c|c|c|c|c|c|c|c|c|}
\hline \multirow{2}{*}{ Variável } & \multicolumn{10}{|c|}{ Ano } & \multirow{2}{*}{ p-valor } & \multirow{2}{*}{$\mathbf{B}^{*}$} \\
\hline & 2011 & 2012 & 2013 & 2014 & 2015 & 2016 & 2017 & 2018 & 2019 & 2020 & & \\
\hline \multicolumn{13}{|c|}{ Vive com HIV } \\
\hline Sim & 9.794 & 9.799 & 9.855 & 10.120 & 9.863 & 9.546 & 9.951 & 9.870 & 9.799 & 8.557 & 0.1362 & -7201 \\
\hline Não & 47.966 & 48.675 & 52.268 & 54.171 & 57.959 & 59.902 & 64.164 & 68.484 & 70.400 & 60.157 & 0.0007 & 2271 \\
\hline \multicolumn{13}{|c|}{ Vive com AlD S } \\
\hline Sim & 8.954 & 8.982 & 8.973 & 9.305 & 9.040 & 8.807 & 9.194 & 9.157 & 8.943 & 7.736 & 0.1839 & -65.94 \\
\hline Não & 61.475 & 61.512 & 62.603 & 63.149 & 65.961 & 67.771 & 72150 & 76.315 & 78.507 & 70.375 & 0.0008 & 1.796 \\
\hline \multicolumn{13}{|c|}{ Uso de tabaco } \\
\hline Sim & 238 & 1.420 & 2.046 & 5.538 & 15.577 & 18.523 & 21.101 & 23.362 & 23.763 & 20.972 & 0.0001 & 3.025 \\
\hline Não & 19.328 & 18.007 & 20.264 & 35.141 & 61.303 & 60.763 & 62976 & 64.977 & 65.427 & 58.540 & 0.0010 & 6.008 \\
\hline \multicolumn{13}{|c|}{ Uso de drogas ilicitas } \\
\hline Sim & 2.059 & 2649 & 3229 & 5.552 & 10.239 & 11.367 & 13.460 & 15.227 & 15.683 & 13.626 & 0.0000 & 1.698 \\
\hline Não & 17.534 & 16.818 & 19.169 & 35.317 & 66.167 & 67.468 & 70.245 & 72.461 & 73.133 & 65.552 & 0.0007 & 7.266 \\
\hline \multicolumn{13}{|c|}{ Possui doença mental } \\
\hline Sim & 1.955 & 1.944 & 1.938 & 1.877 & 2.049 & 2.121 & 2256 & 2.317 & 2.324 & 2.104 & 0.0065 & 43.06 \\
\hline Não & 76.191 & 75.373 & 75.931 & 75.114 & 76.195 & 77.570 & 82225 & 86.198 & 86.946 & 75.715 & 0.0664 & 913.7 \\
\hline \multicolumn{13}{|c|}{ Portador de Diabetes } \\
\hline Sim & 5.476 & 5.607 & 5.796 & 5.582 & 6.000 & 6.273 & 6.656 & 7.198 & 7.664 & 7.371 & 0.0000 & 254.2 \\
\hline Não & 72.710 & 71.703 & 72.093 & 71.516 & 72.559 & 73.681 & 77.987 & 81.663 & 82.084 & 72.495 & 0.0573 & 843.1 \\
\hline \multicolumn{13}{|c|}{ Alcoolismo } \\
\hline Sim & 13.623 & 13.749 & 13.884 & 14.000 & 14.982 & 15.371 & 16.767 & 18.062 & 18.168 & 16.167 & 0.0009 & 505.5 \\
\hline Não & 65.203 & 64.158 & 64.623 & 63.674 & 63.969 & 64.857 & 68.222 & 70.888 & 71.617 & 63.567 & 0.1378 & 505.1 \\
\hline
\end{tabular}

Fonte: Ministério da Saúde/SVS - Sistema de Informação de Agravos de Notificação - Sinan Net

Notas: Os dados foram coletados no dia 8 de setembro de 2021.

Foram considerados, como estatisticamente significativos, os casos em que $\mathrm{p}$-valor $<0.05$

* Indica o coeficiente de variação angular. 
$\mathrm{O}$ presente estudo evidenciou maior incidência dos casos de TB entre o sexo masculino, raça/cor da pele parda, conjuntamente, entre pessoas na faixa etária acima de 55 e mais de 65 anos o que corrobora com a literatura.7,8,9,10

A alta prevalência inclusive de mortalidade por TB para o sexo masculino comumente está relacionada a um maior contato com o bacilo em atividades laborais e a maior frequência de hábitos nocivos à saúde entre os homens.11,8 Mesmo que existam evidências de que as mulheres são mais suscetíveis à progressão da doença, a resposta imunológica pode ser diferente de acordo com o sexo. Todavia, as mulheres apresentam maior índice de cuidado com a saúde em comparação ao sexo masculino.11

A TB apresenta-se como uma doença negligenciada, cujo perfil se associa à vulnerabilidade de ordem social como situação de pobreza, precariedade em saúde e educação. Num país como o Brasil, deve-se considerar a magnitude das desigualdades sociais que tornam vulneráveis os indivíduos à manifestação da doença.12 A vulnerabilidade à $\mathrm{TB}$ pode ser vinculada à comorbidades como diabetes mellitus, infecção por HIV, distúrbios de saúde mental, silicose e condições imunossupressoras crônicas, bem como desnutriçãa, juntamente com abuso de álcool, tabaco e outras drogas. O que são fatores preditivos para a não adesão ao tratamento da doença e aumento dos custos de saúde.4

Ressalta-se que pacientes coinfectados com HIV/AIDS apresentam risco aumentado de morte e que um terço dos óbitos relacionados ao HIV são decorrentes da TB.8
Além disso, a presença de diabetes aumenta a gravidade e o risco de desfechos desfavoráveis no tratamento influenciando na sua gravidade e causando sintomas mais graves da doença como mortalidade por TB. Pessoas com diabetes e TB têm mais dificuldade em controlar os níveis de glicose no sangue do que uma pessoa com diabetes sem TB. 13,12

A população com transtornos mentais e em estado de vulnerabilidade social apresenta maior risco ao desenvolvimento de doenças transmissíveis, como a TB. Dentre as implicações no diagnóstico e tratamento da $\mathrm{TB}$, nesta população, encontra-se a dificuldade de adesão aos tratamentos, maior chance de contrair a TB, multidroga-resistente e baixa qualidade de vida.14 Da mesma maneira, o uso de tabaco, drogas ilícitas e álcool apresentam-se como fatores associados ao reingresso ao tratamento, o que além de prejudicar o sistema imunológico contribui de forma expressiva para o abandono e adesão irregular à quimioterapia.15,8

Ademais, sucedeu uma tendência de aumento dos casos entre pessoa com segundo grau completo de escolaridade. A baixa escolaridade é um preditor de mortalidade por $\mathrm{TB}$, considerada um reflexo de todo um conjunto de circunstâncias precárias, que aumentam a vulnerabilidade à TB e são responsáveis pela maior ocorrência da doença e menor adesão ao tratamento. Em contrapartida, o maior nível de escolaridade tem sido associado a uma menor prevalência de mortalidade por TB.15,16

A estabilidade dos casos da doença, notificados no Brasil, ao longo dos dez anos analisados, sugere-se que a vigilância de casos de TB apresentou alguns avanços. Entretanto, o controle é um desafio para o Brasil, o que mostra a necessidade de maiores investimentos no desenvolvimento de políticas públicas, uma vez que os problemas sociais são reflexos das políticas ineficientes. 16

Fatores como os impedimentos para investigar contatos; fazer um diagnóstico precoce e iniciar a terapia antituberculose na atenção primária; bem como o atraso no diagnóstico e a baixa eficácia da terapia antituberculose, que pode ser decorrente de fatores socioculturais associados ao paciente e a organização do sistema de saúde, favorecem a disseminação da TB.17 A análise espacial de um problema de saúde pode contribuir para a compreensão de sua inserção na sociedade, seus potenciais de enfrentamento e por consequência, a situação de saúde de indivíduos e grupos específicos da população.7

\section{CONCLUSÃO}

Os resultados deste estudo demonstram que houve uma tendência de estabilidade dos casos confirmados e notificados de TB no Brasil. Esses resultados, apesar de sugerirem a melhora das estratégias de vigilância, prevenção e controle da doença, demandam ações intersetoriais de enfrentamento da $\mathrm{TB}$, tendo em vista a tendência de aumento observadas entre homens, pardos, pessoas acima de 55 anos, residentes na região norte do país, bem como o expressivo aumento de casos entre pessoas alcoólicas e diabéticas.

\section{REFERÊNCIAS}

1 Canto VB, Nedel FB. Completude dos registros de tuberculose no Sistema de Informação de Agravos de Notificação (Sinan) em Santa Catarina, Brasil, 2007-2016*. Epidemiologia e Serviços de Saúde, [S.L.], v. 29, n. 3, p. 1-10, jul. 2020. FapUNIFESP (SciELO). http://dx.doi.org/10.5123/ s1679-49742020000300020.

2 Rabelo JVC, Navarro PD, Carvalho WS, Almeida IN, Oliveira CSF, Haddad JPA, Miranda SS. Avaliação do desempenho dos serviços de atenção primária à saúde no controle da tuberculose em metrópole do Sudeste do Brasil. Cadernos de Saúde Pública, [S.L.], v. 37, n. 3, p. 1-13, 2021 FapUNIFESP (SciELO). http://dx.doi.org/10.1590/0102-311x00112020.
3 Pedroso MRO, Guidoni LM, Zandonade E, Fregona G, Negri LSA, Oliveira SMVL. Custos catastróficos e sequelas sociais decorrentes do diagnóstico e tratamento da tuberculose no Brasil. Epidemiologia e Serviços de Saúde, [S.L.], v. 30, n. 3, p. 1-27, 2021. FapUNIFESP (SciELO). http://dx. doi.org/10.1590/s1679-49742021000300012.

4 Moreira ASR, Kritski AL, Carvalho ACC. Social determinants of health and catastrophic costs associated with the diagnosis and treatment of tuberculosis. Jornal Brasileiro de Pneumologia, [S.L.], v. 46, n. 5, p. 1-5, 2020. Sociedade Brasileira de Pneumologia e Tisiologia. http://dx.doi. org/10.36416/1806-3756/e20200015. 


\section{REFERÊNCIAS}

5 Brasil, Ministério da Saúde. Brasil Livre da Tuberculose. Plano Naciona pelo Fim da Tuberculose como Problema de Saúde Pública. Estratégias para 2021-2025. MS. Disponivel em: https://www.gov.br/saude/pt-br/ media/pdf/2021/abril/26/plano-nacional-pelo-fim-da-tb_para-consulta-publica.pdf

6 Souza CSB, Santos GM, Gouvêa AR, Costa MML, Mafra ALS, Pinto Neto JM, Santos EM. O significado da tuberculose pulmonar para a pessoa que adoece e seus comunicantes. SaudColetiv (Barueri) [Internet]. $11^{\circ}$ de maio de 2020 [citado $22^{\circ}$ de setembro de 2021];9(50):1760-8. Disponivel em: http://www.revistas.mpmcomunicacao.com.br/index.php/ saudecoletiva/article/view/153

7 Cavalin RF, Pellini ACG, Lemos RRG, Sato APS. Coinfecção TB-HIV. Revista de Saúde Pública, [S.L.], v. 54, p. 1-13, 14 dez. 2020. Universidade de Sao Paulo, Agencia USP de Gestao da Informacao Academica (AGUIA). http://dx.doi.org/10.11606/s1518-8787.2020054002108.

8 Moraes AP, Soares JPCF, Vieira RFS, Santos DR, Guibilei DB, Carvalho MOP. Notification of 6442 Cases of bone tuberculosis in Brazil: epidemiological profile from 2009 to 2018. Coluna/Columna, [S.L.], v. 20, n. 2, p. 105-108, jun. 2021. FapUNIFESP (SciELO). http://dx.doi.org/10.1590/ s1808-185120212002239569.

9 Silva DJ, Abreu FP, Xavier LEF, Duarte LP, Neves VJR, Souza AA et al. Estudo de série histórica de casos de tuberculose entre 1999 e 2015, em Ouro Preto, Minas Gerais, Brasil. Cadernos Saúde Coletiva, [S.L.], v. 29 , n. 1, p. 36-45, mar. 2021. FapUNIFESP (SciELO). http://dx.doi. org/10.1590/1414-462×202129010120.

10 Rodrigues NCP, Andrade MK de N, O'dwyer G, Flynn M, Braga JU, Almeida AS de, Bastos LS, Lino VTS. Distribution of pulmonary tuberculosis in Rio de Janeiro (Brazil): a spatial analysis. Ciência \& Saúde Coletiva, [S.L.], v. 22, n. 12, p. 4125-4134, dez. 2017. FapUNIFESP (SciELO). http:// dx.doi.org/10.1590/1413-812320172212.0143016.

11 Cecilio HPM, Santos AL, Marcon SS, Latorre MRDO, Mathias TAF, Rossi RM. Tendência da mortalidade por tuberculose no estado do Paraná Brasil - 1998 a 2012. Ciência \& Saúde Coletiva, [S.L.], v. 23, n. 1, p. 241
248, jan. 2018. FapUNIFESP (SciELO). http://dx.doi.org/10.1590/141381232018231.25242015

12 Sousa GGS, Pascoal LM, Costa ACPJ, Santos FS; Santos LH, Arcêncio RA et al. Trend and factors associated with Tuberculosis-Diabetes Mellitus comorbidity in a Northeastern Brazilian municipality. Revista Brasileira de Enfermagem, [S.L.], v. 74, n. 3, p. 1-8, 2021. FapUNIFESP (SciELO). http://dx.doi.org/10.1590/0034-7167-2020-1238.

13 Beraldo AA, Andrade RLP, Pinto ÉSG, Silva-Sobrinho RA, Saita NM, Monroe AA et al. Tuberculosis and diabetes mellitus: sociodemographic and clinical profile in brazilian municipalities. Revista Gaúcha de Enfermagem, [S.L.], v. 45, p. 1-10, 2021.

14 Arroyo LAH, Arcoverde MAM, Alves JD, Fuentealba-Torres M, Cartagena-Ramos D, Scholze AR et al. Spatial analysis of cases of Tuberculosis with Mental Disorders in São Paulo. Revista Brasileira de Enfermagem, [S.L.], v. 72, n. 3, p. 654-662, jun. 2019. FapUNIFESP (SciELO). http://dx. doi.org/10.1590/0034-7167-2017-0949.

15 Silva TC, Matsuoka P da FS, Aquino DMC de, Caldas A de JM. Fatores associados ao retratamento da tuberculose nos municípios prioritários do Maranhão, Brasil. Ciência \& Saúde Coletiva, [S.L.], v. 22, n. 12, p. 40954104, dez. 2017. FapUNIFESP (SciELO). http://dx.doi.org/10.1590/1413812320172212.20612015.

16 Alves JD, Arroyo LH, Arcoverde MAM, Cartagena-Ramos D, Berra TZ, Alves LS et al. Magnitud de los determinantes sociales en el riesgo de mortalidad por tuberculosis en el Centro-Oeste de Brasil. Gaceta Sanitaria, [S.L.], v. 34, n. 2, p. 171-178, mar. 2020. Elsevier BV. http://dx.doi. org/10.1016/j.gaceta.2019.01.004.

17 Mancuzo EV, Netto EM, Sulmonett N, Viana VS, Croda J, Kritski AL et al. Spirometry results after treatment for pulmonary tuberculosis: comparison between patients with and without previous lung disease. Jornal Brasileiro de Pneumologia, [S.L.], v. 46, n. 2, p. 1-9, 2020. Sociedade Brasileira de Pneumologia e Tisiologia. http://dx.doi.org/10.36416/18063756/e20180198. 